\title{
Urban brown rats (Rattus norvegicus) as possible source of multidrug-resistant Enterobacteriaceae and meticillin-resistant Staphylococcus spp., Vienna, Austria, 2016 and 2017
}

Amélie Desvars-Larrive1, Werner Ruppitsch², Sarah Lepuschitz², Michael P Szostak ${ }^{1}$, Joachim Spergser ${ }^{1}$, Andrea T Feßler ${ }^{3}$,

Stefan Schwarz ${ }^{3}$, Stefan Monecke ${ }^{4,5,6}$, Ralf Ehricht ${ }^{4,6}$, Chris Walzer ${ }^{1,7}$, Igor Loncaric ${ }^{1}$

1. University of Veterinary Medicine, Vienna, Austria

2. Austrian Agency for Health and Food Safety, Vienna, Austria

3. Freie Universität, Berlin, Germany

4. Leibniz Institute of Photonic Technology (IPHT), Jena, Germany

5. Technische Universität, Dresden, Germany

6. InfectoGnostics Research Campus, Jena, Germany

7. Wildlife Conservation Society, Bronx, New York, United States

Correspondence: Amélie Desvars-Larrive (amelie.desvars@vetmeduni.ac.at)

Desvars-Larrive Amélie, Ruppitsch Werner, Lepuschitz Sarah, Szostak Michael P, Spergser Joachim, Feßler Andrea T, Schwarz Stefan, Monecke Stefan, Ehricht Ralf, Walzer Chris, Loncaric Igor. Urban brown rats (Rattus norvegicus) as possible source of multidrug-resistant Enterobacteriaceae and meticillin-resistant Staphylococcus spp., Vienna, Austria, 2016 and 2017. Euro Surveill. 2019;24(32):pii=1900149. https://doi.org/10.2807/1560-7917.ES.2019.24.32.1900149

Background: Brown rats (Rattus norvegicus) are an important wildlife species in cities, where they live in close proximity to humans. However, few studies have investigated their role as reservoir of antimicrobialresistant bacteria. Aim: We intended to determine whether urban rats at two highly frequented sites in Vienna, Austria, carry extended-spectrum $\beta$-lactamaseproducing Enterobacteriaceae, fluoroquinoloneresistant Enterobacteriaceae and meticillin-resistant (MR) Staphylococcus spp. (MRS). Methods: We surveyed the presence of antimicrobial resistance in 62 urban brown rats captured in 2016 and 2017 in Vienna, Austria. Intestinal and nasopharyngeal samples were cultured on selective media. We characterised the isolates and their antimicrobial properties using microbiological and genetic methods including disk diffusion, microarray analysis, sequencing, and detection and characterisation of plasmids. Results: Eight multidrug-resistant Escherichia coli and two extensively drug-resistant New Delhi metallo- $\beta$ lactamases-1 (NDM-1)-producing Enterobacter xiangfangensis $\mathrm{ST}_{114}$ (En. cloacae complex) were isolated from nine of 62 rats. Nine Enterobacteriaceae isolates harboured the $b l a_{\text {CTX-M }}$ gene and one carried a plasmid-encoded $a m p C$ gene $\left(b / a_{\mathrm{CMY}-2}\right)$. Forty-four MRS were isolated from 37 rats; they belonged to seven different staphylococcal species: $S$. fleurettii, S. sciuri, S. aureus, S. pseudintermedius, $S$. epidermidis, $S$. haemolyticus (all mecA-positive) and mecC-positive $S$. xylosus. Conclusion: Our findings suggest that brown rats in cities are a potential source of multidrug-resistant bacteria, including carbapenem-resistant En. xiangfangensis ST114. Considering the increasing worldwide urbanisation, rodent control remains an important priority for health in modern cities.

\section{Introduction}

The emergence of multidrug-resistant pathogens that are difficult, and sometimes impossible, to treat is becoming a global concern for human and domestic animal health $[1,2]$. Extended-spectrum $\beta$-lactamase (ESBL)-producing Enterobacteriaceae (ESBL-E), carbapenem-resistant Enterobacteriaceae (CRE) and meticillin-resistant (MR) Staphylococcus aureus (MRSA) are recognised as a threat to healthcare and patient safety [1]. One of the most recently described carbapenemase genes, the New Delhi metallo- $\beta$-lactamase-1 (NDM-1) gene, is located on self-transmissible plasmids that carry several additional antimicrobial resistance genes, which makes NDM-1 a challenge for public health [3]. The role of urban wildlife as reservoirs and/or vectors of antimicrobial resistance (AMR) is poorly understood. Not only do they provide a biological mechanism for the spread of AMR genes [4], they are also considered sentinels of environmental pollution by antimicrobialresistant bacteria [5].

More than half of the world population now is urban and by 2030 , that fraction will have increased to $60 \%$ [6]. Cities can serve as focal points for pathogen introduction and dissemination [7]. The dense human population, increased interactions with urban wildlife and the warmer urban microclimate favour the emergence 
Workflow of the laboratory testing of urban brown rats for antimicrobial-resistant bacteria, Vienna, 13 September 2016-6 June 2017

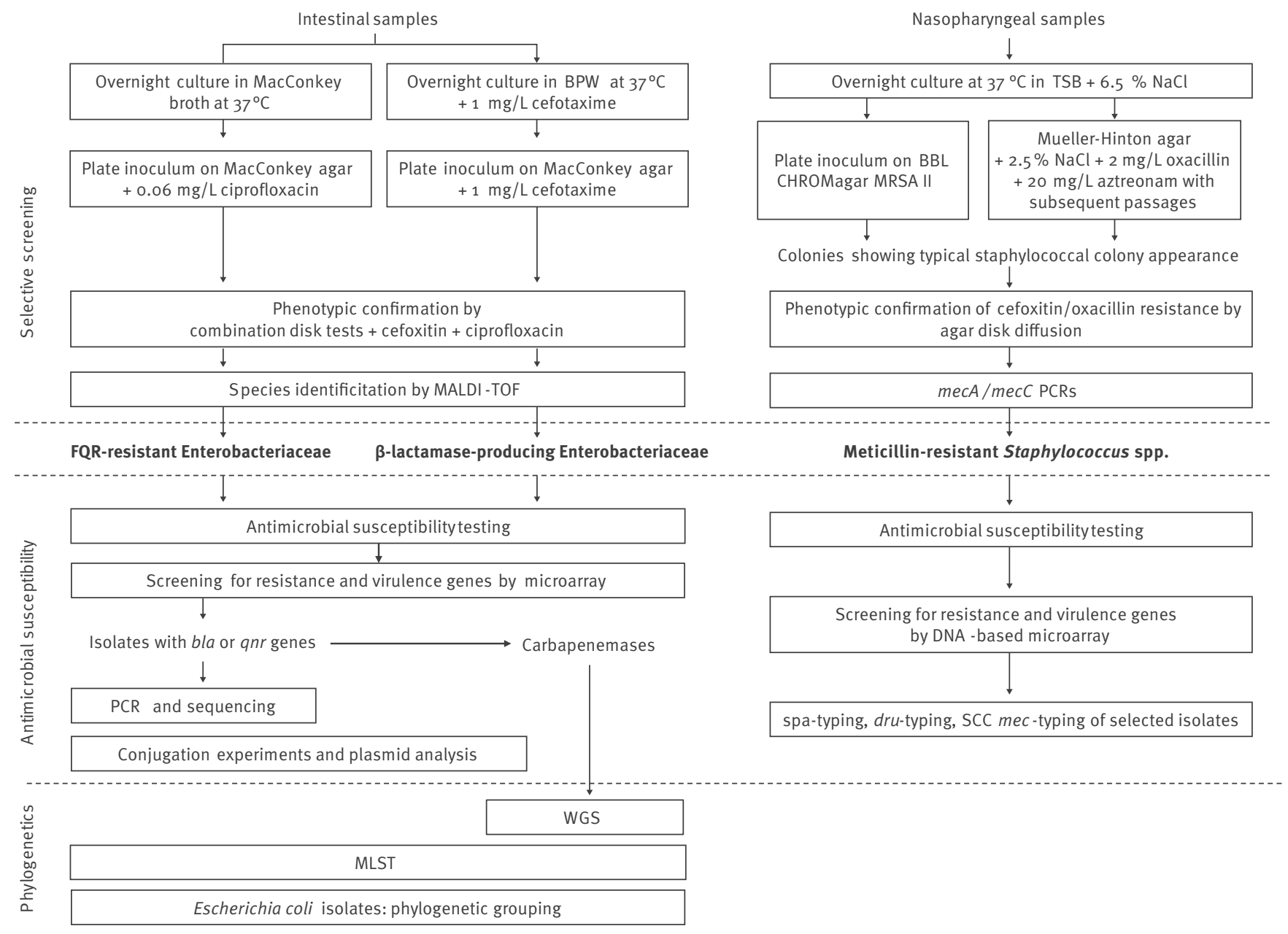

BPW: buffered peptone water; FQR: fluoroquinolone; MALDI-TOF: matrix-assisted laser desorption/ionisation-time-of-flight mass spectrometry; MLST: multilocus sequence typing; MRSA: meticillin-resistant Staphylococcus aureus; TSB: tryptic soy broth; WGS: whole genome sequencing.

of wildlife-borne zoonoses in cities [7]. With regard to AMR, wild brown rats (Rattus norvegicus) are particularly relevant. Rats are considered the most prolific and widespread urban pest species [8]. Synanthropic rats, foraging on human waste and colonising the sewage system, frequently interact with human faeces. Therefore, they are exposed to anthropogenic antimicrobial residues and can acquire, carry and spread multidrug-resistant bacteria [9,10]. However, little is known about their role in the epidemiology of AMR. Antimicrobial-resistant Enterobacteriaceae were isolated from urban rats in Piraeus, Greece [11], Berlin, Germany [9], Hong Kong [12] and Vancouver, Canada [13]. Kato et al. reported carriage of antimicrobialresistant $S$. aureus in urban rats in Tokyo, Japan, although meticillin resistance was not mentioned [14]. Prevalence of MRSA and MR Staphylococcus pseudintermedius (MRSP) in urban rats was investigated in
Vancouver, Canada $[15,16]$ while Hansen et al. described the resistome of urban brown rats in Malaysia, Hong Kong, and Denmark [10].

Because each city presents a specific environmental and socioeconomic context, local studies are needed to evaluate the role of urban rats in the epidemiology of AMR. Our main objective was to determine whether urban rats from two highly frequented places of the city centre of Vienna, Austria, carry ESBL-E, fluoroquinolone-resistant Enterobacteriaceae and MR Staphylococcus spp (MRS).

\section{Methods}

\section{Sampling protocol}

Rattus norvegicus were trapped on 17 occasions between 12 September 2016 and 6 June 2017 in the city 


\begin{tabular}{|c|c|c|c|c|c|c|c|c|c|c|}
\hline 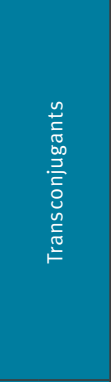 & $\underline{\underline{z}}$ & $\Sigma$ & $\frac{s}{z}$ & $\underline{\underline{z}}$ & & $\underline{z}$ & 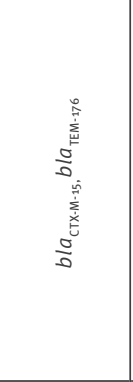 & 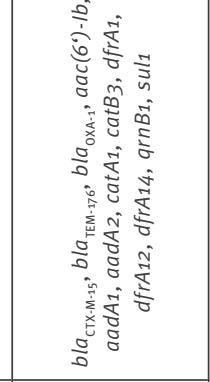 & 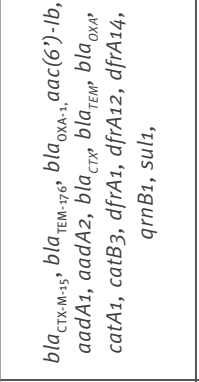 & 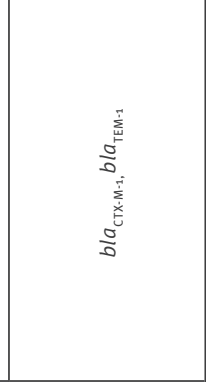 \\
\hline 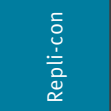 & $\frac{\pi}{z}$ & $\underline{z}$ & $\underline{z}$ & $\frac{\pi}{z}$ & 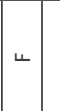 & $\underline{z}$ & 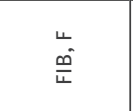 & $\stackrel{\mathbb{I}}{I}$ & $\stackrel{N}{I}$ & $z$ \\
\hline 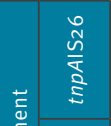 & $\underline{z}$ & $\Sigma$ & $\underline{z}$ & $\frac{s}{z}$ & & $\underline{z}$ & $\underline{z}$ & $\frac{\pi}{z}$ & $\frac{\pi}{z}$ & $\underline{s}$ \\
\hline 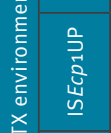 & $\frac{\pi}{z}$ & $\stackrel{\substack{n \\
0}}{0}$ & $\frac{\pi}{z}$ & $\left.\mid \begin{array}{c}\mathfrak{n} \\
\vdots \\
0\end{array}\right]$ & 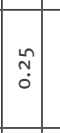 & $\mathbb{s}$ & $\stackrel{\substack{\tilde{O} \\
0}}{ }$ & $\begin{array}{c}\text { â } \\
0\end{array}$ & $\stackrel{\substack{n \\
0}}{0}$ & m \\
\hline 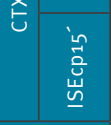 & $\mathbb{z}$ & i & $\mathbb{z}$ & $\frac{\mathbb{z}}{z}$ & \begin{tabular}{|l|l|}
$\frac{s}{z}$ \\
\end{tabular} & $\mathbb{z}$ & $\stackrel{\leftrightarrow}{i}$ & $\stackrel{n}{i}$ & $\stackrel{n}{i}$ & $\stackrel{n}{n}$ \\
\hline 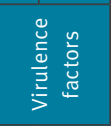 & 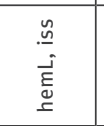 & 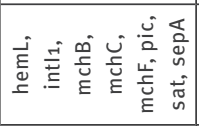 & 咅 & $\frac{s}{z}$ & $\frac{\mathbb{s}}{2}$ & 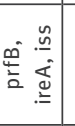 & $\underline{\mathscr{n}}$ & $\frac{\pi}{z}$ & $\frac{\pi}{z}$ & 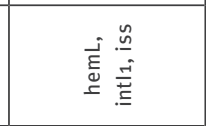 \\
\hline 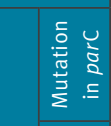 & 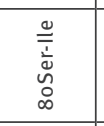 & \begin{tabular}{|l|}
$\sum_{\overline{2}}^{\circ}$ \\
$\frac{0}{3}$ \\
\end{tabular} & 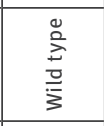 & $\frac{\Sigma}{z}$ & $\frac{\mathbb{z}}{z}$ & $\underline{z}$ & 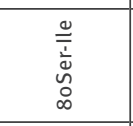 & $\frac{\pi}{z}$ & $\frac{\pi}{z}$ & 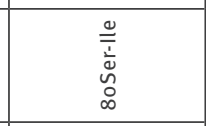 \\
\hline 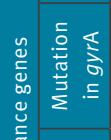 & 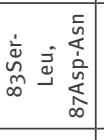 & 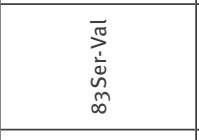 & $\begin{array}{ll}0 \\
\frac{0}{2} \\
\frac{0}{3} \\
\end{array}$ & $\underline{z}$ & \begin{tabular}{|l|l|} 
\\
\end{tabular} & $\frac{\pi}{z}$ & 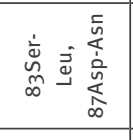 & 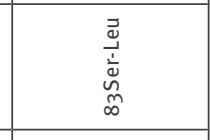 & 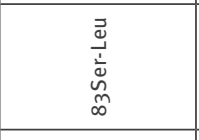 & 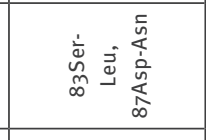 \\
\hline 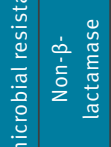 & $\underline{s}$ & 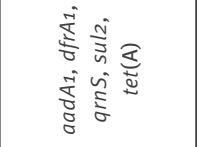 & 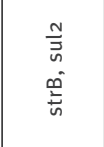 & $\frac{s}{2}$ & $\frac{\pi}{2}$ & 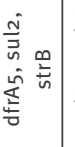 & 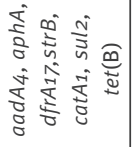 & 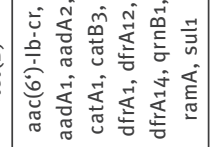 & 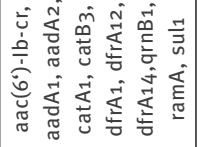 & 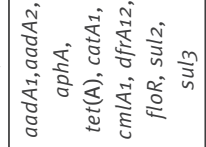 \\
\hline 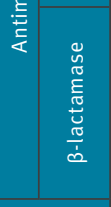 & 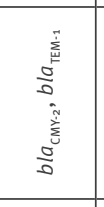 & 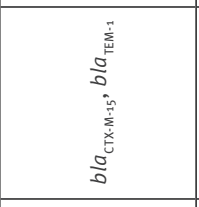 & 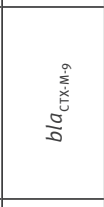 & 豙 & 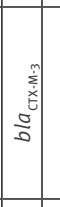 & 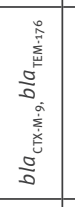 & 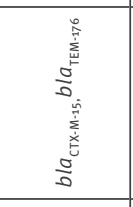 & 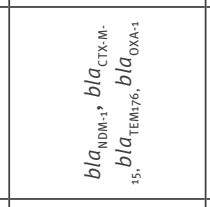 & 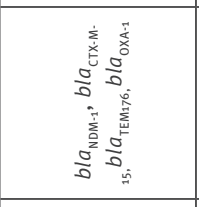 & 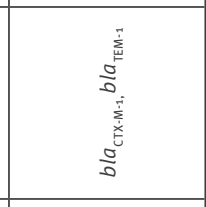 \\
\hline is & 莺 & 旁 & $\stackrel{\infty}{\stackrel{\infty}{\omega}}$ & 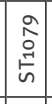 & \begin{tabular}{|l|} 
\\
0 \\
0 \\
\\
5
\end{tabular} & $\stackrel{\substack{n \\
n}}{\stackrel{n}{n}}$ & 蒿 & 志 & 蛋 & 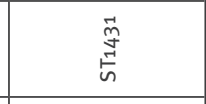 \\
\hline 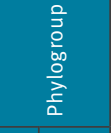 & $\vec{\infty}$ & $u$ & ш & $\vec{\omega}$ & $\vec{\Phi}$ & $\vec{\infty}$ & $u$ & $\frac{\pi}{z}$ & $\frac{\pi}{z}$ & $\vec{\phi}$ \\
\hline 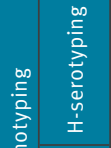 & $\bar{N}$ & $\stackrel{m}{m}$ & $\stackrel{\infty}{\sim}$ & $g$ & g & $\stackrel{\sim}{\sim}$ & $\stackrel{\circ}{m}$ & $\underline{s}$ & $\frac{\pi}{z}$ & $\stackrel{\circ}{m}$ \\
\hline 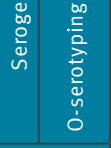 & $\vec{\sim}$ & z & $\stackrel{\infty}{\infty}$ & ० &. & $\infty$ & $a$ & $\frac{\tilde{s}}{z}$ & $\frac{\pi}{z}$ & $\infty$ \\
\hline 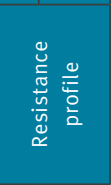 & 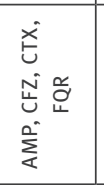 & 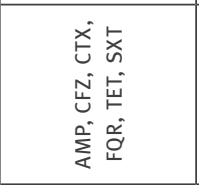 & 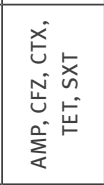 & 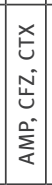 & 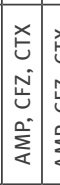 & 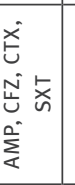 & 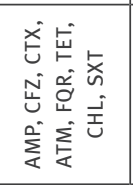 & 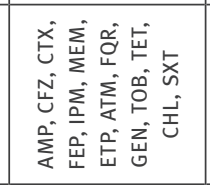 & 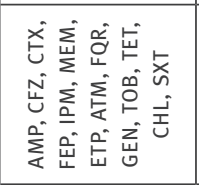 & 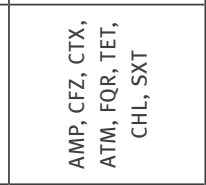 \\
\hline 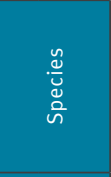 & 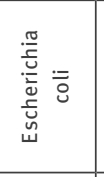 & $\begin{array}{l}\overline{\bar{O}} \\
\dot{u}\end{array}$ & $\begin{array}{l}\overline{\bar{\partial}} \\
\dot{u}\end{array}$ & $\begin{array}{l}\overline{\bar{u}} \\
\dot{u} \\
\end{array}$ & \begin{tabular}{|c|}
$\overline{\bar{o}}$ \\
$\dot{u}$
\end{tabular} & $\begin{array}{l}\overline{\bar{O}} \\
\dot{u} \\
\dot{u}\end{array}$ & $\begin{array}{l}\overline{\bar{o}} \\
\dot{\omega}\end{array}$ & 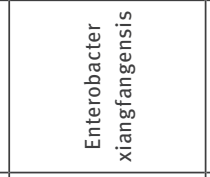 & 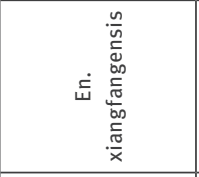 & $\begin{array}{l}\overline{\bar{o}} \\
\text { ن }\end{array}$ \\
\hline 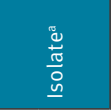 & $\begin{array}{l}\text { ů } \\
\text { 文 }\end{array}$ & $\begin{array}{l}\text { 㟔 } \\
\text { بे }\end{array}$ & $\begin{array}{l}\text { 㟔 } \\
\stackrel{\ddot{\alpha}}{\alpha}\end{array}$ & 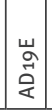 & 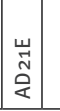 & $\begin{array}{l}\text { J } \\
\text { Ũ⿱亠乂 }\end{array}$ & 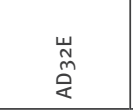 & $\begin{array}{l}\text { 岁 } \\
\text { 定 }\end{array}$ & 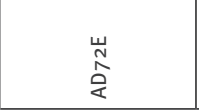 & $\begin{array}{l}\text { 岀 } \\
\text { 定 }\end{array}$ \\
\hline
\end{tabular}


centre of Vienna, Austria: (i) at Karlsplatz (16.37124, 48.20035 decimal degrees (D.D.)), one of the most touristic places in the city, and (ii) at a promenade along the Danube Canal $(48.22631,16.36541$ D.D.). These places were investigated because rats were observed during daytime (whereas $R$. norvegicus is mostly described as a nocturnal species [8]), which suggested an abundant rat population, and because high densities of rats, humans and companion animals may increase contact rates and the risk of pathogen transmission [17].

Rats were captured alive using Manufrance live-traps $(280 \times 100 \times 100 \mathrm{~mm})$. At each location, 20 to 60 traps per night (Karlsplatz: 10 nights, Danube Canal: seven nights) were set between 17.00 and 19.30 and retrieved between 6.00 and 8.00. The trapping effort per site was adjusted according to the method described by Nelson and Clark [18]. Captured animals were transferred alive to the pathology laboratory where morphometric data and samples were collected. Rats were anesthetised using 5\% isoflurane before euthanasia through barbiturate overdose via the intra-peritoneal route. We collected pharyngeal and deep intra-nasal samples using sterile cotton swabs. Swabs were placed individually in Amies transport medium (Heinz Herenz Medizinalbedarf GmbH, Hamburg, Germany) until culture. During necropsy, small intestine and colon tissues were collected aseptically. Freshly collected samples were transported to the Institute of Microbiology in a cooled box.

\section{Characterisation of Enterobacteriaceae isolates}

$\beta$-lactamase-producing Enterobacteriaceae

For each animal, pooled intestinal tissue samples (small intestine and colon) were precultured in buffered peptone water (Merck, Darmstadt, Germany) supplemented with $1 \mathrm{mg} / \mathrm{L}$ cefotaxime and cultivated at $37^{\circ} \mathrm{C}$ overnight on MacConkey agar (Oxoid, Basingstoke, United Kingdom (UK)) supplemented with $1 \mathrm{mg} / \mathrm{L}$ cefotaxime.

Fluoroquinolone-resistant Enterobacteriaceae

In parallel, intestinal samples were cultivated at $37^{\circ} \mathrm{C}$ overnight on MacConkey Broth (Becton Dickinson, Heidelberg, Germany) and cultivated on MacConkey agar supplemented with $0.06 \mathrm{mg} / \mathrm{L}$ ciprofloxacin. For each plate, a single sample of each distinct colony type was picked and regrown on the same medium.

The Enterobacteriaceae isolates were identified by matrix-assisted laser desorption/ionisation-timeof-flight (MALDI-TOF) mass spectrometry (Bruker Daltonik, Heidelberg, Germany). We tested the enterobacterial isolates for production of ESBL using combination disk tests containing cefotaxime and ceftazidime, with or without clavulanic acid (Becton Dickinson, Heidelberg, Germany), according to the Clinical and Laboratory Standards Institute (CLSI) [19]. Furthermore, we used disks containing $30 \mu \mathrm{g}$ cefoxitin and $5 \mu \mathrm{g}$ ciprofloxacin (Becton Dickinson, Heidelberg, Germany) to screen for, respectively, ampicillin C
(AmpC) $\beta$-lactamase-producing and fluoroquinoloneresistant isolates. The ESBL and AmpC phenotypes were confirmed by MASTDISCS ID AmpC and ESBL test (Mast Diagnostics, Merseyside, UK).

We tested the susceptibility of the Enterobacteriaceae isolates against selected antimicrobial agents by the agar disk diffusion method according to the CLSI guidelines [19]. Escherichia coli ATCC 25922 and S. aureus ATCC 25923 served as quality control strains.

We characterised resistance genes using a miniaturised microarray-based assay (CarbDetect-AS-2 Kit, Alere, Jena, Germany). We performed serogenotyping and detected resistance and major virulence genes of $E$. coli isolates using the $E$. coli PanType AS-2 kit (Alere, Jena, Germany). We used PCR to detect bla and $q n r$ genes and then sequenced the amplified products $[20,21]$. Sequences were aligned with BLAST (https://blast.ncbi.nlm.nih.gov/Blast.cgi) and compared with reference sequences available in GenBank and the National Center for Biotechnology Information (NCBI) database (http://www.ncbi.nlm. nih.gov/pathogens/beta-lactamase-data-resources/). Variable regions of class 1 and class 2 integrons were determined by PCR [22]. The quinolone resistance-determining regions (QRDR) of gyrAand parC in ciprofloxacin-resistant isolates were amplified by $P C R$ and sequenced [23]. Using PCR and sequencing, we investigated the genetic environment of bla сTX-M $_{\text {genes, }}$ i.e. the upstream insertion sequences (IS)ECp1 and IS26involved in gene-transferring mechanisms [22,24].

The $E$. coli isolate were assigned to a phylogroup using the quadruplex assignment method [25] and were further subjected to multilocus sequence typing (MLST) [26]. Allelic profiles and sequence types (ST) were determined by querying the $E$. coli MLST website (http:// enterobase.warwick.ac.uk/species/ecoli/allele_st_ search). We performed MLST of the Enterobacter cloacae complex according to the protocol recommended by Miyoshi-Akiyama et al. [27].

We assessed the clonal relatedness of two bla ${ }_{1}$-positive isolates from the En. cloacae complex by whole genome sequencing (WGS). Bacterial DNA was isolated using the MagAttract HMW DNA Kit (Qiagen, Hilden, Germany) and ready-to-sequence libraries were prepared using Nextera XT DNA Library Preparation Kit (Illumina, San Diego, United States). Isolates were paired-end-sequenced using the Illumina MiSeq platform with a read length of $2 \times 300 \mathrm{bp}$ [28]. De novo assembly of raw reads was performed using SPAdes v.3.9.0 [29] and species identification was conducted with the JSpecies workspace using the ANIb (average nucleotide identity via BLAST) analysis tool [30]. We used the Comprehensive Antibiotic Resistance Database (CARD; https://card.mcmaster.ca/home) to identify genes conferring AMR [31]. 
TABLE 2A

Resistance and virulence genetic profile of Staphylococcus spp. isolates from wild urban brown rats, Vienna, Austria, 13 September 2016-6 June $2017(n=44)$

\begin{tabular}{|c|c|c|c|c|c|c|c|c|}
\hline \multirow{2}{*}{ Isolate ${ }^{a}$} & \multirow{2}{*}{ Species $^{b}$} & \multirow{2}{*}{$\begin{array}{c}\text { No. } \\
\text { isolates }\end{array}$} & \multirow{2}{*}{$d r u$} & \multirow{2}{*}{$\mathrm{SCCmec}$} & \multirow{2}{*}{$\mathrm{CC}$} & \multicolumn{2}{|c|}{ Antimicrobial resistance profile } & \multirow{2}{*}{ Virulence factors } \\
\hline & & & & & & Phenotype & Genes detected & \\
\hline AD6 & S. fleurettii & 19 & NA & Irregular & NA & BLA & mecA & NA \\
\hline$A D_{7}$ & S. fleurettii & 19 & NA & Irregular & NA & BLA & mecA & NA \\
\hline AD8 & S. fleurettii & 19 & NA & Irregular & NA & BLA & mecA & NA \\
\hline$A D 9$ & S. fleurettii & 19 & NA & Irregular & NA & BLA & mecA & NA \\
\hline ADgb_MRSP & $\begin{array}{c}\text { S. } \\
\text { pseudintermedius }\end{array}$ & 1 & dt11av & $\mathrm{V}$ & NA & $\begin{array}{l}\text { BLA, TET, } \\
\text { ERY, CLI, } \\
\text { AMK, GEN, } \\
\text { FQR } \\
\end{array}$ & $\begin{array}{c}\operatorname{mec} A, \text { blaz, blal, blaR, } \\
\text { tet }(\mathrm{K}), \text { tet }(\mathrm{M}), \text { erm }(\mathrm{B}), \text { aacA- } \\
\text { aphD, aphA3, sat }\end{array}$ & eno, isdA, lukS (ST22+ST45) \\
\hline$A D 10$ & S. fleurettii (2) ${ }^{c}$ & 1 & NA & Irregular & NA & BLA & mecA & NA \\
\hline $\begin{array}{l}\text { AD1ob_S. } \\
\text { xylosus_ } \\
\text { mecC }\end{array}$ & S. xylosus ${ }^{c}$ & 1 & NA & $\mathrm{XI}$ & NA & BLA & mecC, blaZ-SCCmec XI & NA \\
\hline AD11 & S. fleurettii $(5)^{c}$ & 1 & NA & Irregular & NA & BLA & mecA & NA \\
\hline$A D_{13}$ & S. fleurettii $(6)^{c}$ & 1 & NA & Irregular & NA & BLA & mecA & NA \\
\hline$A D 15$ & S. fleurettii (7) & 1 & NA & Irregular & NA & BLA & mecA & NA \\
\hline$A D 16$ & S. fleurettii & 3 & NA & Irregular & NA & BLA & mecA & ssl10/set4 \\
\hline$A D 16$ & S. fleurettii (11) c & 1 & NA & Irregular & NA & BLA & mecA & ssl10/set 4 \\
\hline$A D 17$ & S. fleurettii & 3 & NA & Irregular & NA & BLA & mecA & ssl10/set 4 \\
\hline$A D 19$ & S. sciuri & 2 & NA & Irregular & NA & $\begin{array}{l}\text { BLA, ERY, } \\
\text { TET }\end{array}$ & $\begin{array}{c}\text { mecA, blaz, blal, blaR, } \\
\text { tet( }(\mathrm{M}), \operatorname{erm}(\mathrm{B})\end{array}$ & NA \\
\hline$A D 23$ & S. fleurettii & 3 & NA & Irregular & NA & BLA & mecA & ssl10/set 4 \\
\hline$A D 24$ & S. fleurettii & 19 & NA & Irregular & NA & BLA & mecA & NA \\
\hline$A D 25$ & S. fleurettii & 19 & NA & Irregular & NA & BLA & mecA & NA \\
\hline$A D 27$ & S. fleurettii (8) ${ }^{c}$ & 1 & NA & Irregular & NA & BLA & mecA & NA \\
\hline$A D 28$ & S. fleurettii (9) ${ }^{c}$ & 1 & NA & Irregular & NA & BLA & mecA & NA \\
\hline$A D_{30}$ & S. fleurettii & 19 & NA & Irregular & NA & BLA & mecA & NA \\
\hline$A D_{31}$ & S. fleurettii & 19 & NA & Irregular & NA & BLA & mecA & NA \\
\hline$A_{32}$ & S. fleurettii & 19 & NA & Irregular & NA & BLA & mecA & NA \\
\hline$A_{33}$ & S. fleurettii (10) ${ }^{c}$ & 1 & NA & Irregular & NA & BLA & mecA & NA \\
\hline$A_{34}$ & S. fleurettii & 1 & NA & Irregular & NA & BLA & mecA & isaB, isd $A$, lukS (ST22 + ST45) \\
\hline$A D_{35}$ & S. fleurettii & 19 & NA & Irregular & NA & BLA & mecA & NA \\
\hline$A_{3} 6$ & S. fleurettii (12) ${ }^{c}$ & 1 & NA & Irregular & NA & BLA & mecA & NA \\
\hline$A D_{37}$ & S. fleurettii (13) ${ }^{c}$ & 1 & NA & Irregular & NA & BLA & mecA & NA \\
\hline $\mathrm{AD}_{37}$ & S. fleurettii $(4)^{c}$ & 1 & NA & Irregular & NA & BLA & mecA & hllII \\
\hline$A_{3} 8$ & S. fleurettii & 19 & NA & Irregular & NA & BLA & mecA & NA \\
\hline$A D_{39}$ & S. fleurettii & 19 & NA & Irregular & NA & BLA & mecA & NA \\
\hline$A D_{50}$ & S. sciuri & 2 & NA & Irregular & NA & $\begin{array}{l}\text { BLA, ERY, } \\
\text { TET }\end{array}$ & $\begin{array}{c}\text { mecA, blaz, blal, blaR, } \\
\text { tet(M), } \operatorname{erm}(\mathrm{B})\end{array}$ & NA \\
\hline$A D_{52}$ & S. fleurettii & 19 & NA & Irregular & NA & BLA & mecA & NA \\
\hline $\mathrm{AD}_{59}$ & S. fleurettii & 1 & NA & Irregular & NA & BLA & mecA & is $a B$, isdA, lukS (ST22+ $\left.\mathrm{ST}_{45}\right)$, sslio/set4 \\
\hline AD61 & S. fleurettii $(3)^{c}$ & 1 & NA & Irregular & NA & BLA & mecA & sslio/set 4 \\
\hline AD68_MRSE & S. epidermidis & 1 & dt10a & $\begin{array}{l}\text { Pseudo } \\
\mathrm{scC} m e c\end{array}$ & NA & $\begin{array}{l}\text { BLA, TET, } \\
\text { ERY, CLI, } \\
\text { AMK, GEN }\end{array}$ & $\begin{array}{c}\text { mecA, blaz, blal, blaR, } \\
\text { tet(K) erm }(\mathrm{C}), d f r A, \\
\operatorname{aacA-aphD}\end{array}$ & NA \\
\hline AD69a & S. fleurettii & 19 & NA & Irregular & NA & BLA & mecA & NA \\
\hline AD6gb & S. fleurettii & 19 & NA & Irregular & NA & BLA & mecA & NA \\
\hline AD70 & S. fleurettii (1) ${ }^{c}$ & 1 & NA & Irregular & NA & BLA & mecA & $e b p S$, is $a B$, isdA, lukS (ST22+ST45) \\
\hline
\end{tabular}

AMK: amikacin; BLA: $\beta$-lactams; CLI: clindamycin; ERY: erythromycin; FQR: fluoroquinolone; GEN: gentamicin; NA: data not available (absent, therefore not amplified, or not typeable); TET: tetracycline.

${ }^{\text {a }}$ Rat ID + isolate identification if several isolates were isolated from the same rat.

${ }^{b}$ Number in bracket refers to the isolate identification in Figure 2.

c SCCmec-typing via array was performed on these isolates. 
Resistance and virulence genetic profile of Staphylococcus spp. isolates from wild urban brown rats, Vienna, Austria, 13 September 2016-6 June $2017(\mathrm{n}=44)$

\begin{tabular}{|c|c|c|c|c|c|c|c|c|}
\hline \multirow{2}{*}{ Isolate ${ }^{\mathrm{a}}$} & \multirow{2}{*}{ Species $^{b}$} & \multirow{2}{*}{$\begin{array}{l}\text { No. } \\
\text { isolates }\end{array}$} & \multirow{2}{*}{$d r u$} & \multirow{2}{*}{$\mathrm{scCmec}$} & \multirow{2}{*}{$\mathrm{CC}$} & \multicolumn{2}{|c|}{ Antimicrobial resistance profile } & \multirow{2}{*}{ Virulence factors } \\
\hline & & & & & & Phenotype & Genes detected & \\
\hline AD70_MRSA & S. aureus ${ }^{c}$ & 1 & dt11a & VT & $\mathrm{CC}_{398}$ & $\begin{array}{l}\text { BLA, TET, } \\
\text { ERY, CLI }\end{array}$ & $\begin{array}{c}\operatorname{mec} A, \\
\operatorname{blaz}, \operatorname{tet}(\mathrm{K}), \operatorname{tet}(\mathrm{M}), \operatorname{erm}(\mathrm{A})\end{array}$ & $\begin{array}{c}\text { aur, bbp, cap5, capH } 5, \text { cap/5, capK5, clfA, clfB, cna, edh, } \\
\text { ebpS, emp, fib (MRSA 252), fnbA, fnbB, hl, hla, hlgA, } \\
\text { lukF, lukS, hlgA, hsdSX-CC15, hysA1 (MRSA252 + RF122) } \\
\text { and/orhysA2 (cons), hysA1 (MRSA252+RF122) and/ } \\
\text { or hysA2 (COL+US30o), hysA2 (COL+US30o+NCTC), icaA, } \\
\text { icaC, icaD, isaB (MRSA 252) isdA, ImrP, lukX, lukY, map, } \\
\text { sdrC, sdrD, setB1, setB2, stB3, setC, set6-var, set11/set2, } \\
\text { sslo2/set7, sslo4/set9, sslo5/set3, sslo7/set1, sslog/set5, } \\
\text { sslio/set4, sspA, sspB, sspP, vwb }\end{array}$ \\
\hline$A D_{71}$ & S. fleurettii & 19 & NA & Irregular & NA & BLA & mecA & NA \\
\hline $\mathrm{AD}_{72}$ & S. fleurettii & 19 & NA & Irregular & NA & BLA & mecA & NA \\
\hline AD74 & S. fleurettii & 19 & NA & Irregular & NA & BLA & mecA & NA \\
\hline AD74_MRSH & S. haemolyticus & 1 & NA & v & NA & $\begin{array}{l}\text { BLA, TET, } \\
\text { ERY, CLI, } \\
\text { GEN }\end{array}$ & $\begin{array}{c}\text { mecA, blaz, blal, } \\
\text { blaR, aacA-aphD, } \\
m p h(\mathrm{C}), \operatorname{msr}(\mathrm{A}), a a d D, \\
a p h A_{3}, \text { sat, qacA }\end{array}$ & isdA, ImrP-variant $s s p P$-variant \\
\hline$A D 76$ & S. fleurettii & 19 & NA & Irregular & NA & BLA & mecA & NA \\
\hline
\end{tabular}

AMK: amikacin; BLA: $\beta$-lactams; CLI: clindamycin; ERY: erythromycin; FQR: fluoroquinolone; GEN: gentamicin; NA: data not available (absent, therefore not amplified, or not typeable); TET: tetracycline.

a Rat ID + isolate identification if several isolates were isolated from the same rat.

${ }^{b}$ Number in bracket refers to the isolate identification in Figure 2.

c SCCmec-typing via array was performed on these isolates.

The presence of plasmids was determined using PlasmidFinder 1.3 available from the Center for Genomic Epidemiology web server (http://www.genomicepidemiology.org/) [32]. We performed conjugation experiments with bla gene-carrying isolates as donors, confirmed and characterised the transconjugants as previously described [20]. We conducted electrotransformation experiments using a GenePulser II (BioRAD, Vienna, Austria). The conjugative and transferable plasmids were subjected to PCR-based replicon typing [33].

Characterisation of Staphylococcus spp. isolates For each animal, pharyngeal and nasal samples were pooled [34], incubated overnight in tryptic soy broth (Becton Dickinson, Heidelberg, Germany) medium with $6.5 \% \mathrm{NaCl}$, then streaked on a BBL CHROMagar MRSA II (Becton Dickinson, Heidelberg, Germany) and MuellerHinton agar (Oxoid, Basingstoke, UK) with $2.5 \% \mathrm{NaCl}$, $2 \mathrm{mg} / \mathrm{L}$ oxacillin and $20 \mathrm{mg} / \mathrm{L}$ aztreonam. For each plate, a single sample of each distinct colony type was picked and regrown on the same medium. Meticillin resistance was confirmed by an agar disk diffusion method with $30 \mu \mathrm{g}$ cefoxitin or $1 \mu \mathrm{g}$ oxacillin (Becton Dickinson, Heidelberg, Germany) [19].

The mecA or mecC genes were amplified by PCR [35] and MRS were characterised to species level by PCR amplification and sequencing of the $r p o B$ gene [36]. Those MR staphylococci harbouring mecA or mecC were tested for susceptibility against selected antimicrobial agents using the agar disk diffusion method according to the CLSI guidelines [19]. Detection of resistance and virulence genes was conducted using the $S$. aureus Genotyping Kit 2.0 DNA microarray (Alere, Jena, Germany) [37]. We further analysed MRSA, MRSP, MR S. epidermidis (MRSE) and MR S. haemolyticus (MRSH) by dru-typing, while MRSA and MRSP were additionally examined by spa-typing [38]. We analysed the staphylococcal cassette chromosome mec (SCCmec) elements of some selected isolates using a DNA-basedmicroarray (Alere, Jena, Germany) [39].

A workflow of the laboratory procedures is provided in Figure 1.

\section{Statistical analyses}

We considered the following explanatory variables: place of capture, body mass (g), body length (nose to anus, mm) and sex. The impact of these factors on the occurrence of AMR (presence of at least one resistant isolate) and on the number of antimicrobialresistant isolates per animal was assessed using, respectively, a generalised linear model under a binomial and a Poisson distribution. The best-fitted model was identified using a stepwise backward selection based on the Akaike Information Criterion. Statistical analyses were conducted using R 3.5.1 (R Development Core Team, Vienna, Austria).

\section{Nucleotide sequence accession numbers}

Because the two En. xiangfangensis isolates are most probably emerging in Austria, it is of public health importance to make their sequences publicly available. Therefore, the genomes of these two isolates were deposited under PRJNA517386 in the NCBI BioProject database. 


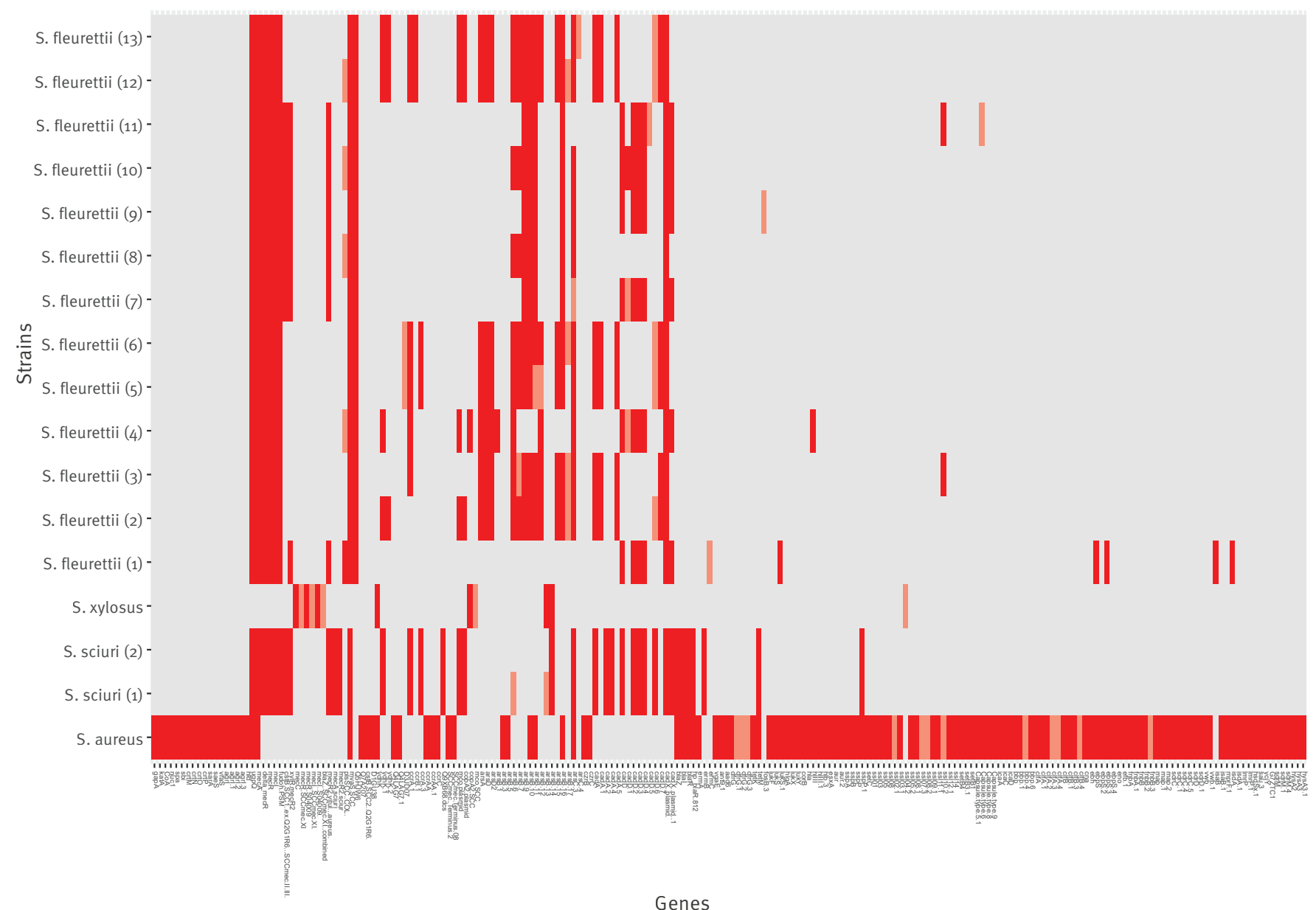

Grey: negative; red: positive; light red: ambiguous.

\section{Ethical statement}

We followed institutional and national standards for the care and use of animals in research. This study was approved by the institutional ethics and animal welfare committee and the national authority (GZ 68.205/0196-WF/V/3b/2016).

\section{Results}

\section{Trapping}

Seventy-six brown rats were captured. Among them, 14 were not further analysed because samples could not be sent fast enough to the Institute of Microbiology after necropsy. Thirty-six rats from Karlsplatz (of which 18 were male) and 26 from Danube Canal (15 males) were ultimately included in the study (Supplementary Figure $\mathrm{S}_{1}$ ). The median body weight and length were $193.5 \mathrm{~g}$ and $196.5 \mathrm{~mm}$ on Karlsplatz, $153.2 \mathrm{~g}$ and 184.0 mm at Danube Canal (Supplementary Table S1).
The adjusted trapping effort was 284.5 trap-nights at Karlsplatz and 238 at Danube Canal.

\section{Characterisation of Enterobacteriaceae isolates}

Ten cefotaxime-resistant Enterobacteriaceae were isolated from the intestinal samples of nine of the 62 rats (Table 1). We identified eight isolates as E. coli and two as members of the En. cloacae complex. Seven serogenotypes were detected among the $E$. coli isolates. All 10 isolates were susceptible to amikacin. Seven $E$. coli isolates displayed the ESBL phenotype. One E. coli isolate and both isolates from the En. cloacae complex displayed the AmpC and ESBL phenotype. All Enterobacteriaceae isolates were ampicillin- and cefazolin-resistant, while four $E$. coli and both isolates from the En. cloacae complex were also tetracyclineand fluoroquinolone-resistant. Both isolates from the En. cloacaecomplex were carbapenem-resistant. Based on Sweeney et al., these two isolates can be considered as extensively drug-resistant (not susceptible 
Relative frequencies of antimicrobial resistance phenotypes at each investigated site in (A) Enterobacteriaceae and (B) Staphylococcus spp. isolates, Vienna, Austria, 13 September 2016-6 June $2017(\mathrm{n}=44)$
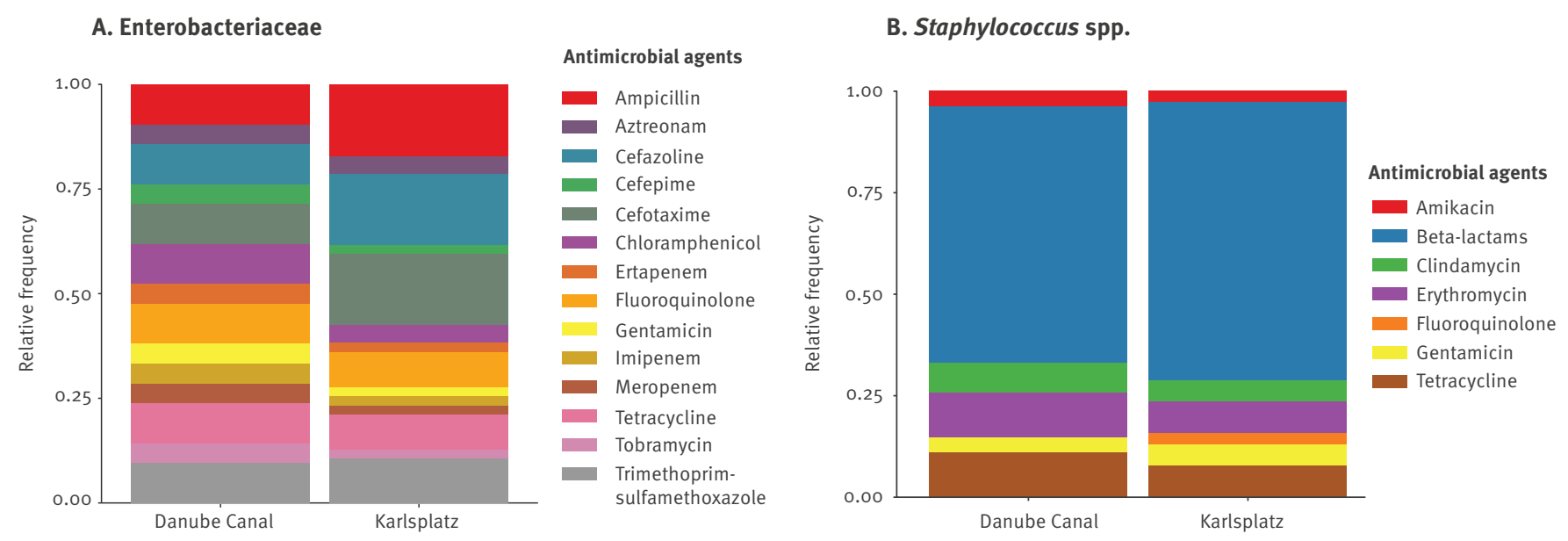

These graphs represent the relative frequency (proportion between o and 1) of AMR phenotype. The sum of the relative frequencies of all AMR phenotypes per site for each bacterial group is 1.0.

to at least one agent in all but one or two available antimicrobial classes) [40].

The $E$. coli isolates belonged to three different phylogroups: B1, C and E. Seven different E. coli ST were detected. MLST identified both members of the En. cloacae complex as ST114. WGS-based ANIb analysis identified these two isolates as En. xiangfangensis carrying the NDM-1 gene on a plasmid similar to type IncH (GenBank accession number: CP016921). PlasmidFinder identified this plasmid as belonging to the IncHl2 group.

In nine of $10 E$. coli isolates, genes from the bla ${ }_{\text {Стх-м }}$ family were detected, alone (three isolates) or in combination with other bla genes (four isolates). The E. coli AD8E isolate harboured the genes bla ${ }_{\text {сMY-2 }}$ and bla ${ }_{\text {TEM-1 }}$. Both En. xiangfangensis isolates carried four bla genes $\left(b_{\mathrm{NDM}-1}\right.$, bla $_{\mathrm{CTX}-\mathrm{M}-15}$, bla $a_{\mathrm{TEM}-176}$, and bla $\left.{ }_{\mathrm{OXA}-1}\right)$.

The genes sul 2 and aad $A 1$ were the most prevalent non$\beta$-lactamase genes detected. A class 1 integron was detected in four ESBL-producing $E$. coli and in both $E n$. xiangfangensis isolates. However, we could not amplify the variable regions of the class 1 integron by PCR. Amino acid substitutions were detected in the QRDR of gyrA and parC. Complete or truncated ISECP1 elements were detected upstream of the bla ${ }_{\text {стх-м }}$ gene in seven isolates, five $E$. coliand both En. xiangfangensis. We did not identify the IS26 element in any isolate. The CTX$\mathrm{M}$-positive isolates showed a 48 -bp region $\mathrm{W}$ sequence upstream of gene bla ${ }_{\text {стх-M-1 }}$, as previously described (GenBank accession number: AM040707), whereas one CTX-M-1-positive isolate harboured the same 48 -bp
W sequence plus a 32-bp $X$ sequence, as previously described (GenBank accession number: AMo03904).

The hem $L$ gene (encoding the glutamate-1-semialdehyde aminotransferase) and the iss (increased serum survival) gene were the most prevalent virulence determinants among the $E$. coli isolates. Transfer of resistance was demonstrated for bla genes by conjugation of three ESBL-producing $E$. coli isolates as donors with either the sodium azide-resistant $E$. coli J53 or the sodium azide- and rifampin-resistant $E$. coli MT 102 as the recipient. Using both En. xiangfangensis isolates as donors, conjugation and transformation of the bla ${ }_{\mathrm{NDM}-1}$ gene were unsuccessful but transfer of the bla ${ }_{\text {СтX-M }}$ genes was achieved.

\section{Characterisation of \\ meticillin-resistant Staphylococcus spp.}

Forty-four MRS belonging to seven species were isolated from 37 of 62 rats (Table 2). Among them, we identified MRSA $(n=1$; prevalence in the rat population: $1.6 \%)$ MRSP $(n=1)$, MRSE $(n=1), \operatorname{MRSH}(n=1)$, MR S. xylosus $(n=1)$ and two species from the $S$. sciuri group, i.e. MR S. fleurettii $(n=37$ isolates; $54.8 \%$ ) and MR S. sciuri $(n=2 ; 3.2 \%)$. All MRS were mecA-positive with the exception of the single MR S. xylosus that was mecC-positive. All MR S. fleurettii and the MR $S$. xylosus were susceptible to all tested non- $\beta$-lactam antimicrobial agents. The other six MR staphylococcal isolates were classified as multidrug-resistant [40].

The MRSA isolate belonged to the clonal complex 398. It belonged to spa type to11, dru type dt11a, and was positive for $a$ - and $\delta$-haemolysin, accessory 
gene regulator (agr) group I and capsule type 5. Its SCCmec type was SCCmec VT + czrC as in strain MRSA ST398/isolate So385 (GenBank accession number: AM990992.1). The MRSP isolate showed spa type to2 and dru type dt11av. The MRSH isolate belonged to SCCmec type $\mathrm{V}$ but the dru sequence could not be amplified. The MRSE isolate belonged to dru type dt1oa and had pseudo SCCmec. Additional SCCmectyping revealed that members of $S$. sciuri group carried different irregular SCCmec elements. The mecC-positive MR S. xylosus isolate carried the type E mec gene complex. The results from SCCmec subtyping are presented in Figure 2.

\section{Epidemiology of antimicrobial resistance}

The overall prevalence of antimicrobial-resistant bacteria in the sampled rats was $62.9 \%$ (39/62). Twelve of the 39 positive rats showed co-colonisation with two (10 rats), three (1 rat) or four (1 rat) antimicrobial-resistant isolates (Supplementary Table S1, Supplementary Figure S1). Seven Enterobacteriaceae-positive rats were also positive for MRS. Enterobacteriaceae (Figure $3 \mathrm{~A}$ ) and Staphylococcus spp. (Figure 3B) isolates from both investigated places shared respectively the same AMR profile, although the relative phenotype frequencies were slightly different and resistance to fluoroquinolone in Staphylococcus spp. was only found at Karlsplatz.

Body weight and length were highly correlated (Spearman's Rho=0.96; $\mathrm{p}<10^{-16}$ ). Therefore, body length was excluded from the models. Occurrence of $A M R$ and number of antimicrobial-resistant isolates per animal were best explained by the single variable body mass although the $p$ values of the coefficient estimates were not significant $(p=0.07$ and $p=0.11$, respectively).

\section{Discussion}

We screened 62 urban brown rats captured in two highly frequented places in the city centre of Vienna, Austria, in 2016 and 2017. We isolated eight multidrug-resistant $E$. coli, two extensively drug-resistant NDM-1-producing En. xiangfangensis ST114 and 44 MRS belonging to seven species. To the best of our knowledge, this is the first isolation of NDM-1-producing strains from the En. cloacae complex in Austria, although NDM-1 was described in the En cloacaecomplex in other countries [41]. The isolate was identified as En. xiangfangensis harbouring simultaneously bla ${ }_{\text {NDM-1 }}$, bla ${ }_{\text {CTX-M- }}$ ${ }_{15}$, a bla ${ }_{\text {TEM }}$-like gene, the plasmid-mediated quinolone resistance gene $q r n B 1$, and diverse other resistance genes. Moreover, this is the first report of MRSE, MRSH, MR mecC-positive $S$. xylosus and MR members of the $S$. sciurigroup in synanthropic brown rats.

The prevalence of multidrug-resistant Enterobacteriaceae in urban brown rats in Vienna, Austria (14.5\% of 62 ) was similar to the prevalence reported in Berlin, Germany, (13.6\%; sampling period 2008-09) [9] and Hong Kong (13.9\%; 2008-13) [12]. In contrast, in Piraeus, Greece, $61.5 \%$ of the urban rats carried multidrug-resistant $E$. coli strains (sampling period not specified) [11] while in Vancouver, Canada, the prevalence was $6.5 \%(2011-12)$ [13]. All Viennese Enterobacteriaceae isolates were resistant to extendedspectrum cephalosporins, with nine of 10 carrying a bla ${ }_{\text {стх-м }}(E S B L)$ gene and one a bla ${ }_{\text {сми-2 }}(\mathrm{AmpC})$ gene. The spread of these genes is strongly facilitated by plasmid-mediated horizontal gene transfer [42]. These findings suggest that rats may play a role in the dissemination of ESBL and AmpC-type $\beta$-lactamases between the human, animal and environmental reservoirs.

To date, two NDM-1-producing Klebsiella pneumoniae have been isolated in Austria, from patients originating from Pakistan and Kosovo*, and one NDM-1producing $E$. coli was isolated from a patient originating from India [43]. Species of the En. cloacae complex have recently emerged as the cause of nosocomial outbreaks and difficult-to-treat bacterial infections in the community, and ST114 clones are considered as clones of high epidemic potential [44]. The early detection of emerging carbapenem resistance in urban wildlife is essential in the surveillance and prevention of AMR outbreaks.

We report a high prevalence of MRS in urban brown rats $(59.7 \%$ of 62 ) together with a high diversity of MR staphylococcal species. MRSA was isolated in 22 of 637 (3.5\%) urban brown rats sampled in 2011-12 in Vancouver, Canada [15], which supports the low MRSA prevalence reported in our study (1.2\%). We characterised one isolate as MRSA CC398 VT spa type to11, dru type dt11a. MRSA CC398 was also identified in wild urban brown rats in Vancouver, Canada, but these strains were slightly different, presenting the spa type to34 [15] which only differs from to11 in the duplication of two repeats (to11: 08-16-02-25-34-24-25 vs to34: o8-16-02-25-02-25-34-24-25) (https://spa. ridom.de/spatypes.shtml). Himsworth et al. evidenced shared MRSA lineages in human, livestock and rats, suggesting that rats can be a source of human and livestock infection [15]. In Austria, MRSA CC398 has been reported in livestock, companion animals [45] and human patients [46].

Himsworth et al. estimated the prevalence of MRSP in urban brown rats from Vancouver, Canada, to be $2.1 \%$ $(5 / 237)$ which is close to the prevalence detected here (1.6\%) [16]. The authors discussed the possibility of MRSP transmission between dogs and rats.

We identified one mecC-positive MR S. xylosus (mecAnegative). This isolate was recently described in Loncaric et al. in a 5-year survey of mecC-positive coagulase-negative staphylococci from 767 wild animals in Austria and was the first report of mecC-positive MR $S$. xylosus in brown rat [47].

Although the interaction between urban wildlife reservoirs of $A M R$ and human health risk remains unclear, the overall prevalence of $A M R$ we observed in the 
sampled rats is of concern. Several rats colonised with multidrug-resistant isolates, including one carbapenem-resistant En. xiangfangensis ST114, were captured at Karlsplatz in a neglected garden used by homeless people as a dormitory in summer. This particular situation enhances the risk of spillover of antimicrobialresistant bacteria. In cities, homeless and marginalised people are the most vulnerable to rodent-borne diseases [48], therefore rodent control remains an important priority for urban health.

We did not find any significant impact of the place of capture or the sex and age (approximated by body weight and length) of the rats on AMR occurrence or on the intensity of co-colonisation. Notably, rat Enterobacteriaceae and staphylococcal strains isolated from two urban sites located ca $3 \mathrm{~km}$ apart shared similar AMR profiles. These findings support the hypothesis that rats potentially acquire antimicrobial-resistant bacteria from the environment (including food waste) and suggest that they may act as a reservoir of AMR in cities. Nevertheless, proving rat-to-human transmission is highly challenging. Twelve animals carried more than one resistant isolates, which indicates a potential role of urban rats as melting pot for horizontal gene transfer between bacterial species acquired from different sources at different times [10]. In the wastewater ecosystem, rats are exposed to AMR through direct contact with antimicrobial-resistant bacteria but also with mobile genetic elements, such as bacteriophages or plasmids, that carry AMR genes $[10,42,49]$.

The principal limitations of our study reside in the low sample size $(n=62)$ and number of sites investigated $(n=2)$. A greater sample size would have increased the accuracy of the prevalence estimation and the power of the statistical analysis, therefore providing a more detailed picture of the epizootiology of AMR in urban rats. A greater number of sites would have provided information on the spatial variability and environmental risk factors of AMR in the city. Furthermore, data on antimicrobial-resistant isolates from domestic animals and human patients in Vienna would have given a more complete overview of the epidemiological situation.

\section{Conclusion}

We demonstrate that urban rats are potential spreaders of $\beta$-lactamase-producing Enterobacteriaceae, including the En. cloacae pandemic ST114 clone, and of MRS, which are considered a major risk to global public health. Because rats are ubiquitous in cities, are in contact with sewage effluents, have a generalist and opportunistic diet, interact frequently with human wastes and have a small home range [8], they are a good candidate sentinel species to detect fine-scale variation in the distribution and sources of AMR [50]. Our results stress the importance of urban wildlife species, such as brown rats, as bio-indicators for AMR surveillance programmes in urban ecosystems. This study underlines the importance of One Health approaches in the global context of AMR.
Note

*This designation is without prejudice to positions on status, and is in line with United Nations Security Council Resolution $1244 / 99$ and the International Court of Justice Opinion on the Kosovo Declaration of Independence.

\section{Acknowledgements}

We thank Diana Suzana Gliga (Veterinary University of Vienna, Austria) and Margaret Odom (Cornell College of Veterinary Medicine, New York, United States) for their assistance with fieldwork. We thank Ines Engelmann (BlinkDx, Jena, Germany), Annett Reissig and Elke Müller (Leibniz Institute of Photonic Technology/InfectoGnostics Research Campus, Jena, Germany) for their excellent technical assistance.

Funding statement: $A D L$ and this research were financially supported by a postdoctoral fellowship provided by the University of Veterinary Medicine Vienna, Austria (FU-282-PDC).

\section{Conflict of interest}

Stefan Monecke was, until November 2018, employee of Alere Technologies (Jena, Germany), the company that manufactured the microarrays used in this study.

\section{Authors' contributions}

Chris Walzer, Igor Loncaric, and Amélie Desvars-Larrive designed the research. Chris Walzer and Amélie DesvarsLarrive obtained funding. Amélie Desvars-Larrive conducted the fieldwork.

Werner Ruppitsch, Sarah Lepuschitz, Michael P Szostak, Joachim Spergser, Andrea T Feßler, Stefan Schwarz, Stefan Monecke, Ralf Ehricht, and Igor Loncaric contributed reagents and analytical tools. Amélie Desvars-Larrive and Igor Loncaric performed the data curation, analysis, and visualisation. Amélie Desvars-Larrive and Igor Loncaric wrote the manuscript.

All authors read and approved the manuscript.

\section{References}

1. Centers for Disease Control and Prevention (CDC). Antibiotic resistance threats in the United States, 2013. Atlanta: CDC; 2013. Available from: https://www.cdc.gov/drugresistance/ biggest threats.html?CDC_AA_refVal=https\%3A\%2F\%2Fwww. cdc.gov\%2Fdrugresistance\%2Fthreat-report-2013\%2Findex. html

2. European Centre for Disease Prevention and Control (ECDC). Antimicrobial resistance surveillance in Europe 2016. Annual Report of the European Antimicrobial Resistance Surveillance Network (EARS-Net). Stockholm: ECDC; 2017. Available from: https://ecdc.europa.eu/en/publications-data/ antimicrobial-resistance-surveillance-europe-2016

3. Walsh TR, Toleman MA. The new medical challenge: why NDM1? Why Indian? Expert Rev Anti Infect Ther. 2011;9(2):137-41. https://doi.org/10.1586/eri.10.159 PMID: 21342058

4. Wu J, Huang Y, Rao D, Zhang Y, Yang K. Evidence for environmental dissemination of antibiotic resistance mediated by wild birds. Front Microbiol. 2018;9:745. https://doi. org/10.3389/fmicb.2018.00745 PMID: 29731740

5. Radhouani H, Poeta P, Gonçalves A, Pacheco R, Sargo R, Igrejas G. Wild birds as biological indicators of environmental pollution: antimicrobial resistance patterns of Escherichia coli and enterococci isolated from common buzzards (Buteo 
buteo). J Med Microbiol. 2012;61(Pt 6):837-43. https://doi. org/10.1099/jmm.0.038364-0 PMID: 22403140

6. World Health Organization and UN-Habitat. Global report on urban health: equitable healthier cities for sustainable development. Geneva: World Health Organization; 2016. Available from: https://apps.who.int/iris/handle/10665/204715

7. Hassell JM, Begon M, Ward MJ, Fèvre EM. Urbanization and disease emergence: dynamics at the wildlife-livestock-human interface. Trends Ecol Evol. 2017;32(1):55-67. https://doi. org/10.1016/j.tree.2016.09.012 PMID: 28029378

8. Feng AYT, Himsworth CG. The secret life of the city rat: a review of the ecology of urban Norway and black rats (Rattus norvegicus and Rattus rattus). Urban Ecosyst. 2014;17(1):149 62. https://doi.org/10.1007/s11252-013-0305-4

9. Guenther S, Bethe A, Fruth A, Semmler T, Ulrich RG, Wieler LH, et al. Frequent combination of antimicrobial multiresistance and extraintestinal pathogenicity in Escherichia coli isolates from urban rats (Rattus norvegicus) in Berlin, Germany. PLoS One. 2012;7(11):e50331. https://doi.org/10.1371/journal. pone.0050331 PMID: 23189197

10. Hansen TA, Joshi T, Larsen AR, Andersen PS, Harms K, Mollerup $S$, et al. Vancomycin gene selection in the microbiome of urban Rattus norvegicus from hospital environment. Evol Med Public Health. 2016;2016(1):219-26. https://doi.org/10.1093/emph/ eowo21 PMID: 27412864

11. Burriel AR, Kritas SK, Kontos V. Some microbiological aspects of rats captured alive at the port city of Piraeus, Greece. Int J Environ Health Res. 2008;18(2):159-64. https://doi. org/10.1080/09603120701358432 PMID: 18365804

12. Ho P-L, Lo W-U, Lai EL, Law PY, Leung SM, Wang Y, et al. Clonal diversity of CTX-M-producing, multidrug-resistant Escherichia coli from rodents. J Med Microbiol. 2015;64(Pt 2):185-90. https://doi.org/10.1099/jmm.0.000001 PMID: 25627207

13. Himsworth CG, Zabek E, Desruisseau A, Parmley EJ, ReidSmith R, Jardine CM, et al. Prevalence and characteristics of Escherichia coli and Salmonella spp. in the feces of wild urban Norway and black rats (Rattus norvegicus and Rattus rattus) from an inner-city neighborhood of Vancouver, Canada. J Wildl Dis. 2015;51(3):589-600. https://doi.org/10.7589/2014-09-242 PMID: 25932669

14. Kato Y, Matsunaga S, Misuna Y, Ushioda H, Yamamoto T, Kaneuchi C. Isolation and characterization of Staphylococcus aureus in rats trapped at restaurants in buildings in downtown Tokyo. J Vet Med Sci. 1995;57(3):499-502. https://doi. org/10.1292/jvms.57.499 PMID: 7548405

15. Himsworth CG, Miller RR, Montoya V, Hoang L, Romney MG, Al-Rawahi GN, et al. Carriage of methicillin-resistant Staphylococcus aureus by wild urban Norway rats (Rattus norvegicus). PLoS One. 2014;9(2):e87983. https://doi. org/10.1371/journal.pone.0087983 PMID: 24498421

16. Himsworth CG, Patrick DM, Parsons K, Feng A, Weese JS. Methicillin-resistant Staphylococcus pseudintermedius in rats. Emerg Infect Dis. 2013;19(1):169-70. https://doi.org/10.3201/ eid1901.120897 PMID: 23260061

17. Bradley CA, Altizer S. Urbanization and the ecology of wildlife diseases. Trends Ecol Evol. 2007;22(2):95-102. https://doi. org/10.1016/j.tree.2006.11.001 PMID: 17113678

18. Nelson L, Clark FW. Correction for sprung traps in catch/effort calculations of trapping results. J Mammal. 1973;54(1):295-8. https://doi.org/10.2307/1378903

19. Clinical and Laboratory Standards Institute (CLSI). Performance standards for antimicrobial susceptibility testing. 27th ed. Wayne: CLSI; 2017. Available from: https://clsi.org/standards/ products/microbiology/documents/m10o/

20. Loncaric I, Stalder GL, Mehinagic K, Rosengarten R, Hoelzl $\mathrm{F}$, Knauer F, et al. Comparison of ESBL--and AmpC producing Enterobacteriaceae and methicillin-resistant Staphylococcus aureus (MRSA) isolated from migratory and resident population of rooks (Corvus frugilegus) in Austria. PLoS One. 2013;8(12):e84048. https://doi.org/10.1371/journal. pone.0084048 PMID: 24391878

21. Nordmann P, Poirel L, Carrër A, Toleman MA, Walsh TR. How to detect NDM-1 producers. J Clin Microbiol. 2011;49(2):718-21. https://doi.org/10.1128/JCM.01773-10 PMID: 21123531

22. Dolejska $M$, Frolkova $P$, Florek $M$, Jamborova I, Purgertova $M$, Kutilova I, et al. CTX-M-15-producing Escherichia coli clone B2-025b-ST131 and Klebsiella spp. isolates in municipal wastewater treatment plant effluents. J Antimicrob Chemother. 2011;66(12):2784-90. https://doi.org/10.1093/jac/dkr363 PMID: 21954457

23. Everett MJ, Jin YF, Ricci V, Piddock LJ. Contributions of individual mechanisms to fluoroquinolone resistance in 36 Escherichia coli strains isolated from humans and animals. Antimicrob Agents Chemother. 1996;40(10):2380-6. https:// doi.org/10.1128/AAC.40.10.2380 PMID: 8891148
24. Eckert C, Gautier V, Arlet G. DNA sequence analysis of the genetic environment of various blaCTX-M genes. I Antimicrob Chemother. 2006;57(1):14-23. https://doi.org/10.1093/jac/ dki398 PMID: 16291869

25. Clermont O, Christenson JK, Denamur E, Gordon DM. The Clermont Escherichia coli phylo-typing method revisited: improvement of specificity and detection of new phylogroups. Environ Microbiol Rep. 2013;5(1):58-65. https://doi. org/10.1111/1758-2229.12019 PMID: 23757131

26. Wirth T, Falush D, Lan R, Colles F, Mensa P, Wieler LH, et al. Sex and virulence in Escherichia coli: an evolutionary perspective. Mol Microbiol. 2006;60(5):1136-51. https://doi.org/10.1111/ j.1365-2958.2006.05172.x PMID: 16689791

27. Miyoshi-Akiyama T, Hayakawa K, Ohmagari N, Shimojima M, Kirikae T. Multilocus sequence typing (MLST) for characterization of Enterobacter cloacae. PLoS One. 2013;8(6):e66358. https://doi.org/10.1371/journal. pone.0066358 PMID: 23776664

28. Lepuschitz S, Huhulescu S, Hyden P, Springer B, Rattei T, Allerberger $\mathrm{F}$, et al. Characterization of a community-acquiredMRSA USA 300 isolate from a river sample in Austria and whole genome sequence based comparison to a diverse collection of USA30o isolates. Sci Rep. 2018;8(1):9467. https://doi. org/10.1038/s41598-018-27781-8 PMID: 29930324

29. Bankevich A, Nurk S, Antipov D, Gurevich AA, Dvorkin M, Kulikov AS, et al. SPAdes: a new genome assembly algorithm and its applications to single-cell sequencing. J Comput Biol. 2012;19(5):455-77. https://doi.org/10.1089/cmb.2012.0021 PMID: 22506599

30. Richter M, Rosselló-Móra R, Oliver Glöckner F, Peplies J. JSpeciesWS: a web server for prokaryotic species circumscription based on pairwise genome comparison. Bioinformatics. 2016;32(6):929-31. https://doi.org/10.1093/ bioinformatics/btv681 PMID: 26576653

31. Jia B, Raphenya AR, Alcock B, Waglechner N, Guo P, Tsang KK, et al. CARD 2017: expansion and model-centric curation of the comprehensive antibiotic resistance database. Nucleic Acids Res. 2017;45(D1):D566-73. https://doi.org/10.1093/nar/ gkw1004 PMID: 27789705

32. Carattoli A, Zankari E, García-Fernández A, Voldby Larsen M, Lund $\mathrm{O}$, Villa $\mathrm{L}$, et al. In silico detection and typing of plasmids using PlasmidFinder and plasmid multilocus sequence typing. Antimicrob Agents Chemother. 2014;58(7):3895-903. https:// doi.org/10.1128/AAC.02412-14 PMID: 24777092

33. Carattoli A, Bertini A, Villa L, Falbo V, Hopkins KL, Threlfall EJ. Identification of plasmids by PCR-based replicon typing. J Microbiol Methods. 2005;63(3):219-28. https://doi org/10.1016/j.mimet.2005.03.018 PMID: 15935499

34. Senn L, Basset P, Nahimana I, Zanetti G, Blanc DS. Which anatomical sites should be sampled for screening of methicillin-resistant Staphylococcus aureus carriage by culture or by rapid PCR test? Clin Microbiol Infect. 2012;18(2):E313. https://doi.org/10.1111/j.1469-0691.2011.03724.X PMID: 22192160

35. Loncaric I, Kübber-Heiss A, Posautz A, Stalder GL, Hoffmann D, Rosengarten R, et al. Characterization of methicillin-resistant Staphylococcus spp. carrying the mecC gene, isolated from wildlife. J Antimicrob Chemother. 2013;68(10):2222-5. https:// doi.org/10.1093/jac/dkt186 PMID: 23674764

36. Mellmann A, Becker K, von Eiff C, Keckevoet U, Schumann $P$, Harmsen D. Sequencing and staphylococci identification. Emerg Infect Dis. 2006;12(2):333-6. https://doi.org/10.3201/ eid1202.050962 PMID: 16494767

37. Monecke S, Coombs G, Shore AC, Coleman DC, Akpaka P, Borg $M$, et al. A field guide to pandemic, epidemic and sporadic clones of methicillin-resistant Staphylococcus aureus. PLoS One. 2011;6(4):e17936. https://doi.org/10.1371/journal. pone.0017936 PMID: 21494333

38. Harmsen D, Claus H, Witte W, Rothgänger J, Claus H, Turnwald $\mathrm{D}$, et al. Typing of methicillin-resistant Staphylococcus aureus in a university hospital setting by using novel software for spa repeat determination and database management. I Clin Microbiol. 2003;41(12):5442-8. https://doi.org/10.1128/ JCM.41.12.5442-5448.2003 PMID: 14662923

39. Monecke S, Slickers P, Gawlik D, Müller E, Reissig A, RuppeltLorz A, et al. Molecular typing of ST239-MRSA-III from diverse geographic locations and the evolution of the SCCmec III element during its intercontinental spread. Front Microbiol. 2018;9:1436. https://doi.org/10.3389/fmicb.2018.01436 PMID: 30087657

40. Sweeney MT, Lubbers BV, Schwarz S, Watts JL. Applying definitions for multidrug resistance, extensive drug resistance and pandrug resistance to clinically significant livestock and companion animal bacterial pathogens. J Antimicrob Chemother. 2018;73(6):1460-3. https://doi.org/10.1093/jac/ dky043 PMID: 29481657 
41. Annavajhala MK, Gomez-Simmonds A, Uhlemann A-C. Multidrug-resistant Enterobacter cloacae complex emerging as a global, diversifying threat. Front Microbiol. 2019;10(44):44. https://doi.org/10.3389/fmicb.2019.00044 PMID: 30766518

42. Rozwandowicz M, Brouwer MSM, Fischer J, Wagenaar JA, Gonzalez-Zorn B, Guerra B, et al. Plasmids carrying antimicrobial resistance genes in Enterobacteriaceae. J Antimicrob Chemother. 2018;73(5):1121-37. https://doi. org/10.1093/jac/dkx488 PMID: 29370371

43. Zarfel G, Hoenigl M, Würstl B, Leitner E, Salzer HJF, Valentin T, et al. Emergence of carbapenem-resistant Enterobacteriaceae in Austria, 2001-2010. Clin Microbiol Infect. 2011;17(11):E58. https://doi.org/10.1111/j.1469-0691.2011.03659.x PMID: 21939472

44. Izdebski R, Baraniak A, Herda M, Fiett J, Bonten MJM, Carmeli $Y$, et al. MLST reveals potentially high-risk international clones of Enterobacter cloacae. J Antimicrob Chemother. 2015;70(1):48-56. https://doi.org/10.1093/jac/dku359 PMID: 25216820

45. Loncaric I, Künzel F, Licka T, Simhofer H, Spergser J, Rosengarten R. Identification and characterization of methicillin-resistant Staphylococcus aureus (MRSA) from Austrian companion animals and horses. Vet Microbiol. 2014;168(2-4):381-7. https://doi.org/10.1016/j. vetmic.2013.11.022 PMID: 24332703

46. Krziwanek K, Metz-Gercek S, Mittermayer H. Methicillinresistant Staphylococcus aureus $\mathrm{ST} 398$ from human patients, upper Austria. Emerg Infect Dis. 2009;15(5):766-9. https://doi. org/10.1111/j.1469-0691.2007.01896.x PMID: 18070133

47. Loncaric I, Kübber-Heiss A, Posautz A, Ruppitsch W, Lepuschitz S, Schauer B, et al. Characterization of mecC gene-carrying coagulase-negative Staphylococcus spp. isolated from various animals. Vet Microbiol. 2019;230:138-44. https://doi. org/10.1016/j.vetmic.2019.02.014 PMID: 30827379

48. Leibler JH, Zakhour CM, Gadhoke P, Gaeta JM. Zoonotic and vector-borne infections among urban homeless and marginalized people in the United States and Europe, 19902014. Vector Borne Zoonotic Dis. 2016;16(7):435-44. https:// doi.org/10.1089/vbz.2015.1863 PMID: 27159039

49. Lood R, Ertürk G, Mattiasson B. Revisiting antibiotic resistance spreading in wastewater treatment plants - Bacteriophages as a much neglected potential transmission vehicle. Front Microbiol. 2017;8:2298. https://doi.org/10.3389/ fmicb.2017.02298 PMID: 29209304

50. Furness LE, Campbell A, Zhang L, Gaze WH, McDonald RA. Wild small mammals as sentinels for the environmental transmission of antimicrobial resistance. Environ Res. 2017;154(Supplement C):28-34. https://doi.org/10.1016/j. envres.2016.12.014 PMID: 28013185

\section{License, supplementary material and copyright}

This is an open-access article distributed under the terms of the Creative Commons Attribution (CC BY 4.0) Licence. You may share and adapt the material, but must give appropriate credit to the source, provide a link to the licence and indicate if changes were made.

Any supplementary material referenced in the article can be found in the online version.

This article is copyright of the authors or their affiliated institutions, 2019. 